Chronic Obstructive Pulmonary Diseases: Journal of the COPD Foundation

Original Research

\title{
Identifying Patients with Undiagnosed COPD in Primary Care Settings: Insight from Screening Tools and Epidemiologic Studies
}

MeiLan K. Han, MD, MS ${ }^{1}$ Anna W. Steenrod, $\mathrm{MPH}^{2}$ Elizabeth D. Bacci, $\mathrm{PhD}^{2}$ Nancy K. Leidy, $\mathrm{PhD}^{2}$ David M. Mannino, MD ${ }^{3}$ Byron M. Thomashow, MD ${ }^{4}$ R. G. Barr, MD ${ }^{4}$ Barry J. Make, MD ${ }^{5}$ Russ P. Bowler, MD 5 Stephen I. Rennard, $\mathrm{MD}^{6}$ Julia F. Houfek, $\mathrm{PhD}^{6}$ Barbara P. Yawn, MD, MSc ${ }^{7}$ Catherine A. Meldrum, $\mathrm{PhD}^{1}$ John W. Walsh ${ }^{8}$ Fernando J. Martinez, MD, MS ${ }^{9}$ for the High-Risk-COPD Screening Study Group ${ }^{10}$

\section{Abstract}

Objective: Chronic obstructive pulmonary disease (COPD) is a major cause of morbidity and mortality, yet research suggests this disease is greatly underdiagnosed. This literature review sought to summarize the most common and significant variables associated with case-finding or missed cases of COPD to inform more effective and efficient detection of high-risk COPD patients in primary care.

Methods: PubMed and EMBASE were searched for articles describing case-finding and epidemiologic research to detect or characterize new cases of COPD. International studies in primary and non-primary care settings, published in English from 2002-2014, were eligible for inclusion. Studies related to risk factors for development of COPD were excluded.

Results: Of the 33 studies identified and reviewed, 21 were case-finding or screening and 12 were epidemiological, including cross-sectional, longitudinal, and retrospective designs. A range of variables were identified within and across studies. Variables common to both screening and epidemiological studies included age, smoking status, and respiratory symptoms. Seven significant predictors from epidemiologic studies did not appear in screening tools. No studies targeted discovery of higher risk patients such as those with reduced lung function or risks for exacerbations.

Conclusion: Variables used to identify new cases of COPD or differentiate COPD cases and non-cases are wideranging, (from sociodemographic to self-reported health or health history variables), providing insight into important factors for case identification. Further research is underway to develop and test the best, smallest variable set that can be used as a screening tool to identify people with undiagnosed, high-risk COPD in primary care.

\footnotetext{
Abbreviations: acute exacerbation of COPD, AECOPD; area under the curve, AUC; body mass index, BMI; COPD Assessment Test, CAT; COPD Diagnostic Questionnaire, CDQ; COPD Population Screener Questionnaire, COPD-PS; forced expiratory volume in 1 second, FEV $_{1}$; forced vital capacity, FVC; Global initiative for chronic Obstructive Lung Disease, GOLD; Lung Function Questionnaire, LFQ; Third National Health and Nutrition Examination Study, NHANES III; receiver operating characteristic, ROC; shortness of breath, SOB

Funding Support: Funding for this work was provided by the National Heart, Lung, and Blood Institute, NHLBI: R01 HL 114055 . Project Officer: Antonello Punturieri, MD, PhD.

Date of Acceptance: December 4, 2014

Citation: Han MK, Steenrod AW, Bacci ED, et al for the High-Risk-COPD Screening Study Group. Identifying patients with undiagnosed COPD in primary care settings: Insight from screening tools and epidemiologic studies. J COPD F. 2015; 103-121. doi:http://dx.doi. org/10.15326/jcopdf.2.2.2014.0152
}

This article has an online data supplement. 
1 University of Michigan, Ann Arbor

2 Evidera, Bethesda, Maryland

3 University of Kentucky, Lexington

4 Columbia University, New York, New York

5 National Jewish Health, Denver, Colorado

6 University of Nebraska, Omaha

7 Olmsted Medical Center, Rochester, Minnesota

8 COPD Foundation, Washington, DC

9 Weill Cornell Medical Center, New York, New York

10 High-Risk-COPD Screening Study Group:

Rebecca Copeland, BS, University of Kentucky; Tim Dorius, MD, University of Nebraska Medical Center; David Hengerer, BA, Evidera; Patricia Jellen, RN, MSN, New York Presbyterian Hospital; Katherine Kim, MPH, Evidera; Marge Kurland, RN, Olmsted Medical Center; Karen Malley, BA, Evidera; Lindsey Murray, MPH, Evidera; Jason Shiffermiller, MD, MPH, University of Nebraska Medical Center; Christina Schnell, BA, CCRC, National Jewish Health; Sonja Stringer, MPH, Evidera; Deb Sumnick, PBT, University of Nebraska; Randel Plant, COPD Foundation; Jennifer Underwood, CCRP, National Jewish Health; Beth Whippo, MSN, RN, New York Presbyterian Hospital

\section{Address correspondence to:}

MeiLan K. Han, MD, MS

University of Michigan

Telephone: 734-936-5201

Email: mrking@umich.edu

\section{Keywords:}

chronic obstructive pulmonary disease; primary care; screening literature review

\section{Introduction}

According to the updated 2013 Global initiative for chronic Obstructive Lung Disease (GOLD) guidelines, chronic obstructive pulmonary disease (COPD) is defined as: "a common preventable and treatable disease...characterized by persistent airflow limitation that is usually progressive and associated with an enhanced chronic inflammatory response in the airways and the lungs to noxious particles or gases." 1 Symptoms are primarily chronic and include dyspnea, cough, and sputum production. ${ }^{2,3}$

COPD is the cause of substantial morbidity and mortality worldwide and was recorded as the third leading cause of death in the United States in 2008. ${ }^{4-6}$ Data from the 2011 Behavioral Risk Factor Surveillance System survey reported that an estimated 15 million adults in the United States have been told by a health care provider that they have COPD (age-adjusted prevalence: $6.0 \%){ }^{7}$ Risk factors for developing COPD include, gender, socio-economic factors, aging, infections, as well as tobacco smoke, occupational dust, vapors, fumes, indoor air pollutants, outdoor air pollutants, and genetic factors. ${ }^{8}$

Research also suggests that COPD is greatly underdiagnosed, as indicated by data from the Third National Health and Nutrition Examination Study (NHANES III). These data suggest that over 63\% of adults with evidence of impaired lung function have never been diagnosed with a lung disease (asthma, chronic bronchitis, or emphysema). ${ }^{9}$ Other lines of research have also demonstrated that many cases are first diagnosed at the time of an acute exacerbation of COPD (AECOPD). ${ }^{10}$ Earlier detection of previously undiagnosed, yet clinically significant, COPD in primary care settings could improve short- and longterm patient outcomes and may be cost-effective. ${ }^{11}$ Therapeutic options exist to treat COPD, with the greatest therapeutic benefit likely to be in symptomatic individuals with a forced expiratory volume in 1 second $\left(\mathrm{FEV}_{1}\right)<60 \%$ predicted who are symptomatic or at risk for AECOPD (i.e., clinically significant COPD). ${ }^{12,13}$

Although spirometry is the diagnostic gold standard, ${ }^{1}$ it is not efficient as a case-finding tool, and routine use in primary care is not feasible or cost-effective. A brief, easy-to-use self-administered questionnaire may be a more practical method for identifying people most likely to have clinically significant COPD and who are in need of follow-up and diagnostic spirometric testing. Several screening questionnaires have been developed for use in varied settings, including the general population, primary care, and specialty areas, to identify people with COPD. None of these instruments (e.g., COPD Diagnostic Questionnaire $[\mathrm{CDQ}]^{14}$; COPD Assessment Test $[\mathrm{CAT}]^{15}$ ) have specifically attempted to identify previously undiagnosed individuals with clinically significant COPD or who are at high risk of an exacerbation.

The current project is part of a larger study using a multi-method approach to develop a simple, standardized case-finding method for identifying individuals with moderate to severe airway obstruction 
and those at risk for AECOPD, who have not been diagnosed or treated for COPD. The approach includes a comprehensive literature review, systematic analyses of 3 datasets using random forests methodology, and qualitative research with individuals from the target population.

The purpose of this literature review was to glean insight from existing screening or case-finding instruments and epidemiologic literature related to COPD case identification. Specifically, the intent was to compare and contrast the content and performance of screening questionnaires, as well as identify factors from the epidemiological literature that were predictive of those at high risk of AECOPD, in order to identify

\section{Figure 1. Article Selection Process}

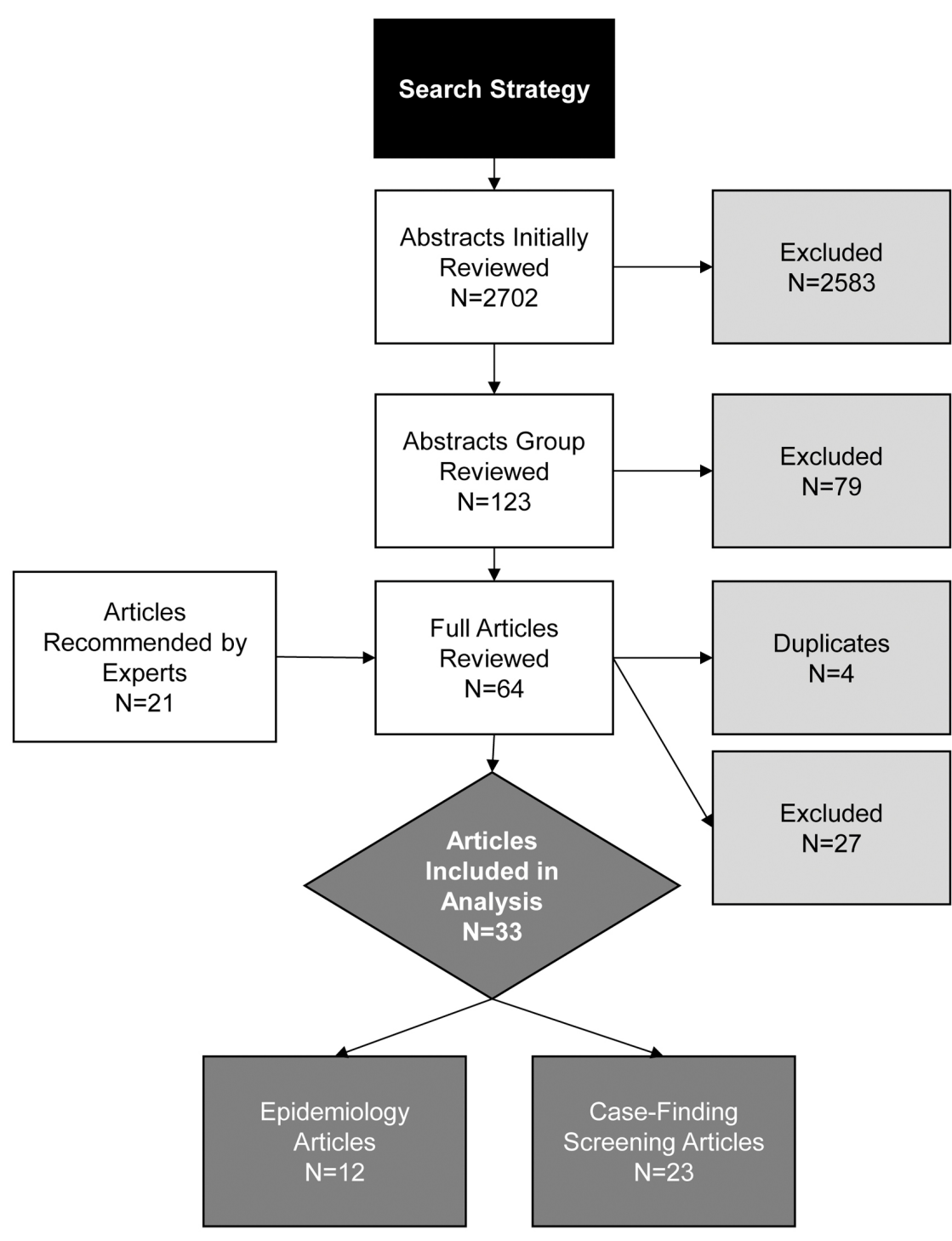

For personal use only. Permission required for all other uses. significant factors, or sets of factors, which would inform the selection of candidate constructs for the new casefinding tool.

\section{Methods}

\section{Search Strategy}

This search used PubMed and EMBASE databases to identify articles published in English, from January 1, 2002 to July 14, 2014. An overview of the search strategy is shown in Figure 1 . The initial step was a broad search, to identify as many candidate articles as possible. Key search terms included: chronic obstructive pulmonary disease, chronic bronchitis, pulmonary emphysema, and related terms in combination with screening, case-finding, undiagnosed, early diagnosis, or questionnaire-specifying adults only. Search terms excluded papers related to depression, anxiety, nutrition, or diet, common but unrelated topics in the COPD and COPD epidemiology literature. Since the goal was to be more expansive than restrictive and review studies across available literature, articles were not restricted by study population (i.e., primary care, general population, etc.)

Abstracts were reviewed by 2 research team members and selected for further review if the study addressed new case identification using screening or epidemiologic methods, and included empirical evidence of screening effectiveness (screening instrument studies) or predictive power (epidemiology studies). Abstracts of studies describing the prevalence of COPD; risk factors for the development of COPD; characteristic features or treatment outcomes of COPD, such as symptoms, quality of life, management strategies, smoking, or smoking cessation; studies using spirometric or genetic testing only; and case studies were excluded from further review.

Abstracts of the selected papers were reviewed by the remaining team members who rated each on a 6-point Likert rating scale of relevance to the identification of 
candidate factors for identifying undiagnosed cases of $\operatorname{COPD}(0=$ not very relevant to $5=$ very relevant $)$. Abstracts rated relevant or very relevant (mean score 24) were selected for full-text review. Team members were also asked to suggest additional articles that should be retrieved for further review.

Two authors examined each of the full-text articles for consistency with the inclusion/exclusion criteria; articles failing to meet these criteria were excluded from further review. Data from the final set of articles were extracted and summarized in tables that included study design, sample size, study population, predictive factors, and results from the statistical analysis, including screening questionnaire performance or the value of predictive factors in identifying new cases of COPD.

\section{Results}

Figure 1 details the results from the article selection process. Of the 2702 abstract reviews and additional 21 papers identified, 64 articles were examined in detail and 33 met the inclusion/exclusion criteria for data extraction. The articles reviewed included 21 casefinding/screening and 12 epidemiologic studies.

\section{Case-finding/Screening Questionnaire Studies}

The case-finding/screening studies included development studies, validation studies, and retrospective data analysis reviews of developed screeners. The median sample size was 676 (range: 39-7701). Studies were conducted in a range of countries: United States ( $n=9)$, Australia $(n=2)$, United Kingdom ( $n=2)$, Netherlands ( $n=1)$, Japan $(n=1)$, Canada $(n=1)$, China $(n=1)$, Greece $(n=1)$, Mexico $(n=1)$, and United States and United Kingdom combined samples $(n=2)$. The study populations consisted of primary care $(n=9)$, the general population ( $n=9)$ (i.e., recruitment through random-digit dialing, conference attendance, and mailed surveys), specialist clinics ( $n=2)$, or a combination of both primary care and specialist clinics $(n=1)$. Most studies $(n=17)$ used the GOLD criteria for COPD case definition, where COPD was defined by a post bronchodilator $\mathrm{FEV}_{1}$ /forced vital capacity (FVC) ratio less than $70 \%{ }^{1} ; 2$ studies used physician diagnosis via medical chart; and 1 study used pre-bronchodilator $\mathrm{FEV}_{1} / \mathrm{FVC}<70 \%$.

Table 1 summarizes key features of the 21 casefinding/screening questionnaires, grouped according to study population (i.e., general population, primary care, specialist, and combined).

The number of questions present in each screener ranged from $2^{23}$ to $10^{26}$ and included a range of factors. Most included age ( $n=20)$, smoking pack years $(n=17)$, phlegm or sputum $(n=16)$, cough $(n=15)$, wheeze $(n=13)$, and dyspnea or shortness of breath $(\mathrm{SOB})(\mathrm{n}=10)$. While these questions were more commonly found across screeners, questions inquiring about exposure $(n=3)$ and family history $(\mathrm{n}=1)$ were also found, highlighting the range of questions that have been used to screen for COPD cases.

Performance characteristics of the screening instruments are summarized in Table 2. All studies reported the sensitivity and specificity of the screener, the majority reported positive and negative predictive values, and approximately half presented results from the area under the receiver operating characteristic (ROC) curve. For the studies conducted in the general population, sensitivities ranged from $65.8 \%$ to $91.7 \%$ and specificity from $46.7 \%$ to $97.7 \%$ (area under the curve $[A U C]=0.65$ to 0.79 ). In primary care studies, sensitivities ranged from $50 \%$ to $91 \%$ and specificities from $25 \%$ to $77 \%$ ( $A U C=0.65$ to 0.85 ). The 1 study that combined primary care and specialist populations (e.g., nursing home) reported sensitivity and specificity within the range of primary care and general population studies (84.4\% and 60.7\%, respectively; AUC=0.73). ${ }^{33}$ The 2 studies utilizing only specialist populations reported the highest sensitivities and good specificities: $90.6 \%$ sensitivity and $77.8 \%$ specificity was reported for the nursing home population ( $\mathrm{AUC}=0.903$ ), ${ }^{34}$ and $92 \%$ sensitivity and $79.4 \%$ specificity was reported for the pulmonary hospital population. 35

\section{Epidemiological Studies}

Table 3 presents an overview of the 12 epidemiological studies, grouped by study population ( 6 general population and 6 primary care). Median sample size was 2578 (range: 146-5002). Studies were conducted in a range of countries: Netherlands $(n=3)$, Norway $(n=2)$, Denmark $(n=2)$, United States $(n=1)$, Canada $(n=1)$, Greece $(n=1)$, and multiple European countries $(n=2)$. The majority $(n=8)$ used the GOLD guidelines for COPD case definition, where COPD was defined by a post bronchodilator FEV1/FVC ratio less than $70 \%{ }^{1}$

Overall, the epidemiological articles assessed 24 predictive factors. Of these, 17 were found to be statistically significant in at least 1 study and 6 were significant in more than half of the studies where 


\section{Table 1. Case-finding/Screening Studies: Design and Factors/Predictors}

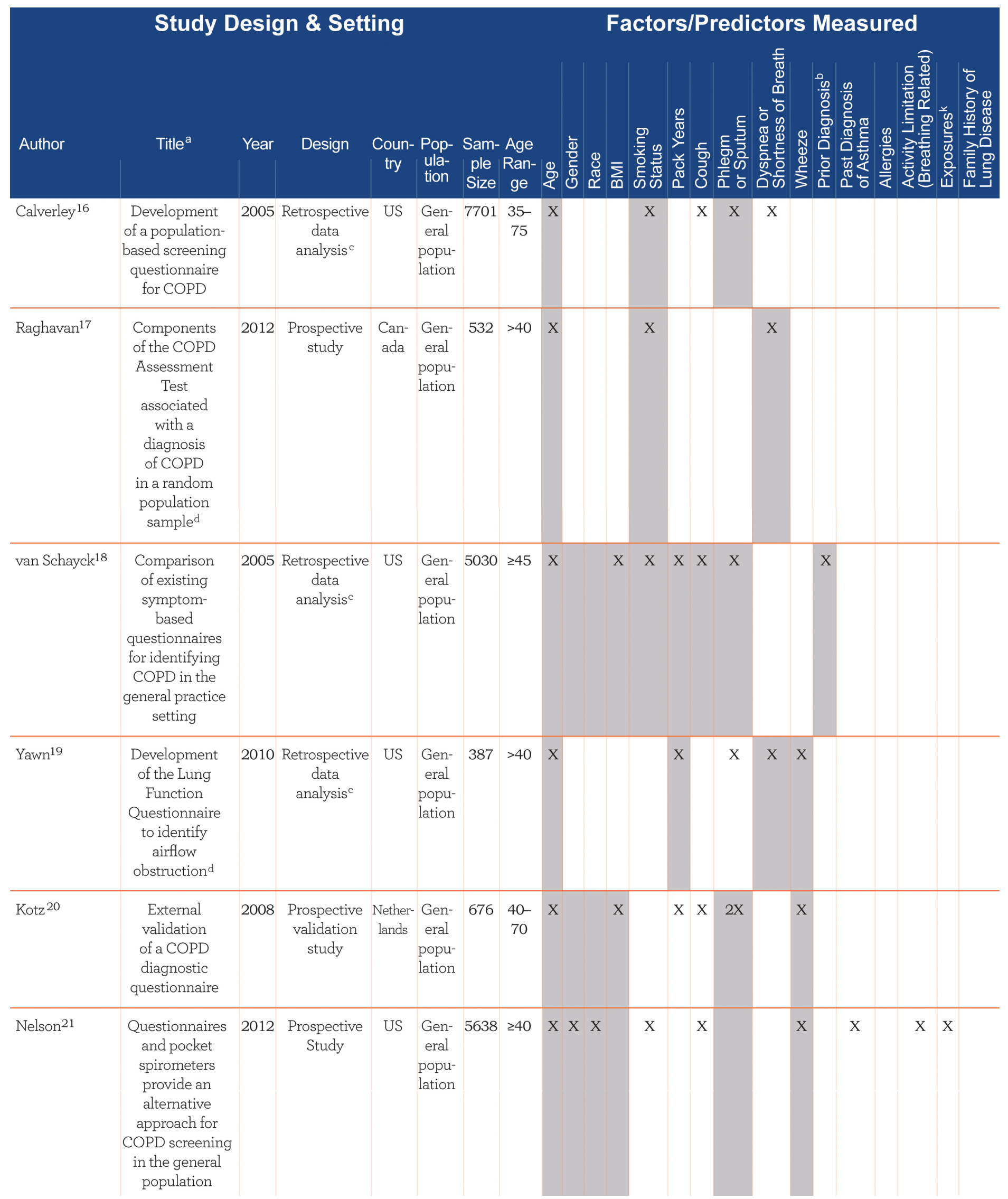




\section{Study Design \& Setting}

\section{Factors/Predictors Measured}

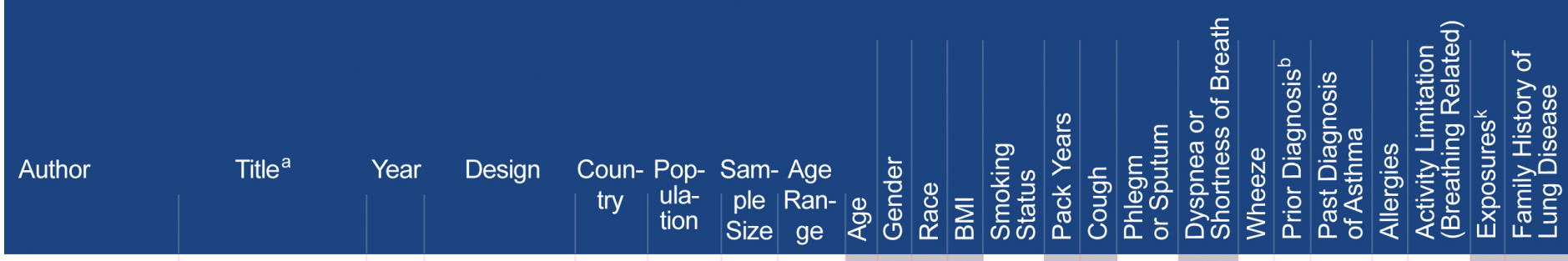

\begin{tabular}{|c|c|c|c|c|c|c|c|c|c|c|c|c|c|c|c|}
\hline Zhou 22 & $\begin{array}{l}\text { Development } \\
\text { and validation } \\
\text { of a chronic } \\
\text { obstructive } \\
\text { pulmonary } \\
\text { disease screening } \\
\text { questionnaire } \\
\text { in China }\end{array}$ & 2013 & $\begin{array}{l}\text { Cross- } \\
\text { sectional } \\
\text { study }\end{array}$ & $\begin{array}{c}\text { Chi- } \\
\text { na }\end{array}$ & $\begin{array}{l}\text { Gen- } \\
\text { eral } \\
\text { popu- } \\
\text { lation }\end{array}$ & 3231 & $\geq 40$ & $\mathrm{X}$ & $\mathrm{X}$ & $\mathrm{X}$ & $\mathrm{X}$ & & & & $\mathrm{X}$ \\
\hline $\begin{array}{l}\text { Franco- } \\
\text { Marina }^{23}\end{array}$ & $\begin{array}{c}\text { Efficient } \\
\text { screening for } \\
\text { COPD using } \\
\text { three steps: } \\
\text { a cross- } \\
\text { sectional study } \\
\text { in Mexico City }\end{array}$ & 2014 & $\begin{array}{c}\text { Retrospective } \\
\text { data } \\
\text { analysis } \\
j\end{array}$ & $\begin{array}{l}\text { Mex- } \\
\text { ico }\end{array}$ & $\begin{array}{l}\text { Gen- } \\
\text { eral } \\
\text { popu- } \\
\text { lation }\end{array}$ & 414 & $\geq 40$ & $\mathrm{X}$ & & $\mathrm{X}$ & $\mathrm{X}$ & $\mathrm{X}$ & $\mathrm{X}$ & $\mathrm{X}$ & $\mathrm{X}$ \\
\hline
\end{tabular}

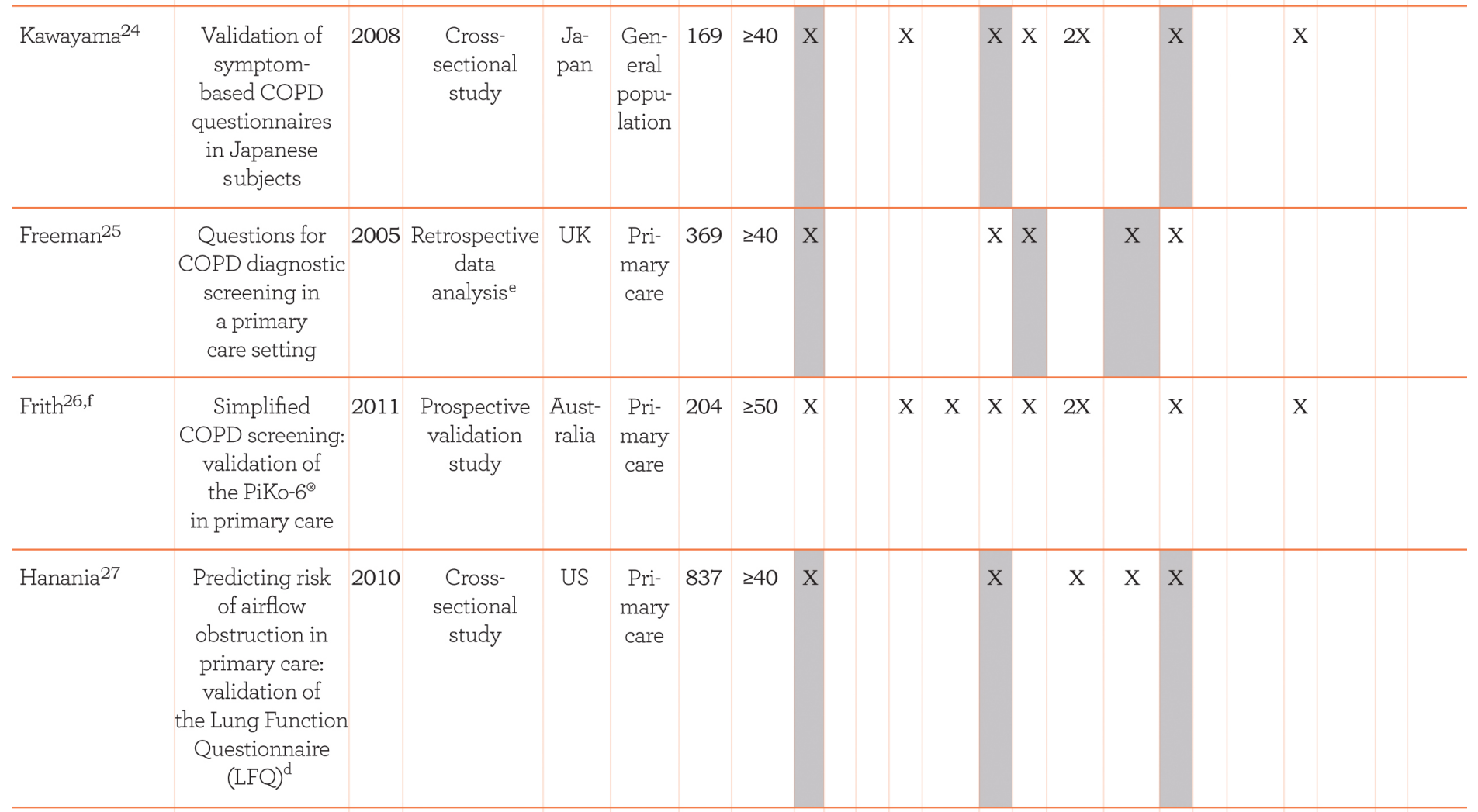

$\operatorname{Mintz}{ }^{28, f}$

Prevalence of

2011 Prospective US Pri- $1575 \geq 30$ X

$\mathrm{X}$ airway

obstruction assessed by mary

Lung Function

Questionnaire

\begin{tabular}{|c|c|c|c|}
$\begin{array}{c}\text { cross- } \\
\text { sectional study }\end{array}$ & $\begin{array}{c}\text { mary } \\
\text { care }\end{array}$ & \\
& & \\
& & \\
& & \\
& & \\
\end{tabular}


Study Design \& Setting

Factors/Predictors Measured

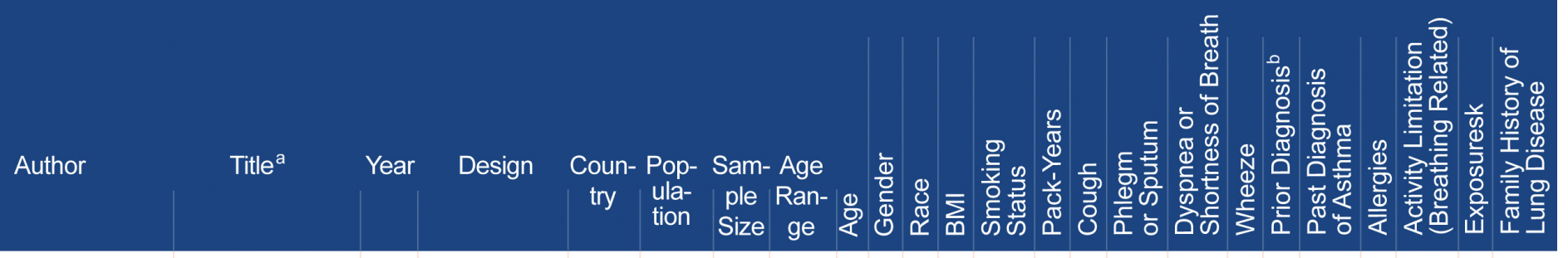

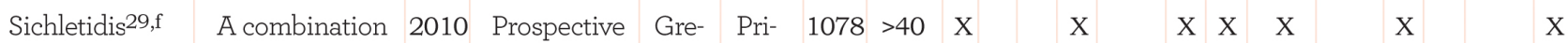
\begin{tabular}{l|l|l} 
of the IPAG & study ece mary
\end{tabular}

questionnaire

and PiKo-6

flow meter

is a valuable

screening tool

for COPD

in the primary

care setting

\begin{tabular}{|c|c|c|c|c|c|c|c|c|c|c|c|c|c|c|c|c|c|}
\hline Price 30 & $\begin{array}{l}\text { Scoring system } \\
\text { and clinical } \\
\text { application of } \\
\text { COPD } \\
\text { diagnostic } \\
\text { questionnaires }\end{array}$ & 2006 & $\begin{array}{c}\text { Primary } \\
\text { care }\end{array}$ & $\begin{array}{l}\text { US \& } \\
\text { Scot- } \\
\text { land }\end{array}$ & $\begin{array}{l}\text { Pri- } \\
\text { mary } \\
\text { care }\end{array}$ & 798 & $\geq 40$ & $X$ & $\mathrm{X}$ & & $\mathrm{X}$ & $\mathrm{X}$ & $2 X$ & & $\mathrm{X}$ & $\mathrm{X}$ & \\
\hline Price $^{14}$ & $\begin{array}{c}\text { Symptom- } \\
\text { based } \\
\text { questionnaire } \\
\text { for identifying } \\
\text { chronic obstructive } \\
\text { pulmonary disease } \\
\text { in smokers }\end{array}$ & 2006 & $\begin{array}{c}\text { Primary } \\
\text { care }\end{array}$ & $\begin{array}{l}\text { US \& } \\
\text { Scot- } \\
\text { land }\end{array}$ & $\begin{array}{l}\text { Pri- } \\
\text { mary } \\
\text { care }\end{array}$ & 818 & $\geq 40$ & $\mathrm{X}$ & $\mathrm{X}$ & & $\mathrm{X}$ & $\mathrm{X}$ & $2 X$ & & $\mathrm{X}$ & $\mathrm{X}$ & \\
\hline Estes $^{31}$ & $\begin{array}{l}\text { An evidence- } \\
\text { based quality } \\
\text { improvement } \\
\text { perspective for } \\
\text { a chronic } \\
\text { obstructive } \\
\text { pulmonary } \\
\text { disease case- } \\
\text { finding program }\end{array}$ & 2014 & $\begin{array}{c}\text { Primary } \\
\text { care }\end{array}$ & US & $\begin{array}{l}\text { Pri- } \\
\text { mary } \\
\text { care }\end{array}$ & 39 & $>40$ & $\mathrm{X}$ & $\mathrm{X}$ & & $\mathrm{X}$ & $\mathrm{X}$ & $2 X$ & & $\mathrm{X}$ & $\mathrm{X}$ & \\
\hline Stanley ${ }^{32}$ & $\begin{array}{l}\text { Validation of } \\
\text { the COPD } \\
\text { Diagnostic } \\
\text { Questionnaire } \\
\text { in an Australian } \\
\text { general practice } \\
\text { cohort: a cross- } \\
\text { sectional study }\end{array}$ & 2014 & $\begin{array}{c}\text { Primary } \\
\text { care }\end{array}$ & $\begin{array}{l}\text { Aus- } \\
\text { tralia }\end{array}$ & $\begin{array}{l}\text { Pri- } \\
\text { mary } \\
\text { care }\end{array}$ & 1054 & $\begin{array}{c}40- \\
85\end{array}$ & $\mathrm{X}$ & $\mathrm{X}$ & & $\mathrm{X}$ & $\mathrm{X}$ & $2 X$ & & $\mathrm{X}$ & $\mathrm{X}$ & \\
\hline Martinez 33 & $\begin{array}{c}\text { Development } \\
\text { and initial } \\
\text { validation of a } \\
\text { self-scored COPD } \\
\text { Population Screener } \\
\text { Questionnaire } \\
\text { (COPD-PS) }\end{array}$ & 2008 & $\begin{array}{c}\text { Prospective } \\
\text { development } \\
\text { study }\end{array}$ & US & $\begin{array}{l}\text { Pri- } \\
\text { mary } \\
\text { care } \\
\text { and } \\
\text { pulm- } \\
\text { onary } \\
\text { spe- } \\
\text { cialist }\end{array}$ & 295 & $\geq 35$ & $\mathrm{X}$ & & $\mathrm{X}$ & & & $\mathrm{X}$ & $\mathrm{X}$ & & & $\mathrm{X}$ \\
\hline
\end{tabular}




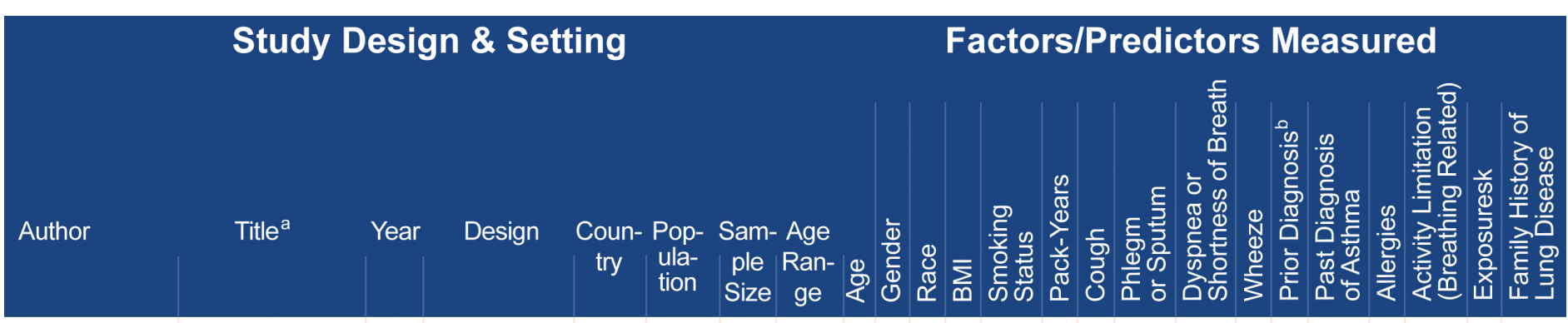

\begin{tabular}{|c|c|c|c|c|c|c|c|c|c|c|c|c|c|c|c|c|}
\hline Zarowitz $34, \mathrm{f}$ & $\begin{array}{l}\text { Development } \\
\text { and validation } \\
\text { of a screening } \\
\text { tool for chronic } \\
\text { obstructive } \\
\text { pulmonary } \\
\text { disease in } \\
\text { nursing home } \\
\text { residents }^{g}\end{array}$ & 2011 & $\begin{array}{c}\text { Retrospective } \\
\text { data } \\
\text { analysish }^{\text {h }}\end{array}$ & US & $\begin{array}{c}\text { Spe- } \\
\text { cialist: } \\
\text { nur- } \\
\text { sing } \\
\text { home }\end{array}$ & 129 & $\geq 65$ & & & $\mathrm{X}$ & & & $2 \mathrm{X}$ & & $\mathrm{X}$ & \\
\hline Müllerová35,f & $\begin{array}{l}\text { Validation of } \\
\text { a chronic } \\
\text { obstructive } \\
\text { pulmonary } \\
\text { disease } \\
\text { screening } \\
\text { questionnaire } \\
\text { for population } \\
\text { surveys }^{\text {i }}\end{array}$ & 2004 & $\begin{array}{l}\text { Prospective } \\
\text { validation } \\
\text { study }\end{array}$ & UK & $\begin{array}{c}\text { Spe- } \\
\text { cialist: } \\
\text { pulm- } \\
\text { onary } \\
\text { hos- } \\
\text { pital }\end{array}$ & 104 & $\geq 45$ & $\mathrm{X}$ & $\mathrm{x}$ & $\mathrm{X}$ & $\mathrm{X}$ & $\mathrm{X}$ & $\mathrm{X}$ & $5 X$ & $\mathrm{X}$ & \\
\hline $\begin{array}{l}{ }^{a} \text { COPD cas } \\
\text { b Prior diag } \\
\text { chronic br } \\
\text { c NHANES } \\
\text { d Postbronc } \\
\text { physician } \\
\text { e Database } \\
\text { f Study did } \\
\text { g COPD cas } \\
\text { h Nursing h } \\
\text { i Questionn } \\
\text { j Mexico Ci } \\
\text { k Exposure: }\end{array}$ & $\begin{array}{l}\text { definition unle } \\
\text { sis of: van Schy } \\
\text { chitis; Franco- } \\
\text { taset used in a } \\
\text { dialator and a } \\
\text { agnosis of asth } \\
\text { lected from } 19 \\
\text { t assess the str } \\
\text { inding: a diagr } \\
\text { le patient regis } \\
\text { re case definiti } \\
\text { PLATINO Sur } \\
\text { lou: biomass fu }\end{array}$ & $\begin{array}{l}\text { s othe } \\
\text { ack-a } \\
\text { Marina } \\
\text { lalysis } \\
\text { iistory } \\
\text { na or } \\
97 \text { to } \\
\text { ength } \\
\text { osis of } \\
\text { try } \\
\text { n of C } \\
\text { ey ( } 20 \\
\text { el fro }\end{array}$ & $\begin{array}{l}\text { erwise noted } \\
\text { asthma, emph } \\
\text { a: Tuberculos } \\
\mathrm{s} \\
\mathrm{y} \text { of at least } 1 \text { ( } \\
\text { other lung di } \\
2002 \text { from } \\
\text { of individual } \\
\text { f COPD } \\
\text { COPD: } 45 \text { ye } \\
\text { O03) and Me } \\
\text { m cooking; } \mathrm{F}\end{array}$ & $\begin{array}{l}\text { is; } \\
\text { o pac } \\
\text { sease } \\
\text { prin } \\
\text { facto }\end{array}$ & $\begin{array}{l}\text { ke years } \\
\text { nary ca } \\
\text { ors in th at le } \\
\text { City Re }\end{array}$ & $\begin{array}{l}\text { s or } 1 \\
\text { rre cli } \\
\text { he an }\end{array}$ & $\begin{array}{l}\text { yea } \\
\text { aic in } \\
\text { lysis }\end{array}$ & ho & $2111 y$ & & $\mathrm{~m}$ & 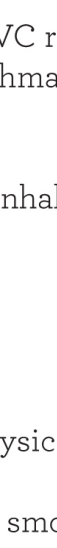 & . & $\begin{array}{l}70 \\
\text { emp } \\
\text { tance } \\
\text { nosis }\end{array}$ & 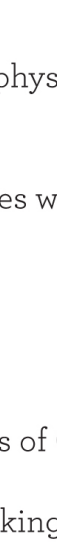 & $\begin{array}{l}\text { a, an } \\
\text { no } \\
\text { PD }\end{array}$ \\
\hline
\end{tabular}

Grey Shading $=$ Odds ratio was significant at $p<0.05$

assessed. Significant factors included: smoking history or status ( $n=8$ of 10 studies), age ( $n=7$ of 10 studies), cough ( $n=5$ of 9 studies), wheeze ( $n=5$ of 7 studies), dyspnea/SOB ( $\mathrm{n}=4$ of 9 studies), gender ( $\mathrm{n}=4$ of 9 studies), phlegm/sputum ( $\mathrm{n}=4$ of 6 studies), and body mass index (BMI) ( $\mathrm{n}=3$ of 5 studies).

\section{Cross-study Comparison}

Similarities and differences in predictive factors identified in the screening and epidemiologic literature are summarized in Table 4.

Although the themes/content categories were similar across study type, the range of factors was broader in the epidemiological literature. Almost half of predictive factors tested in the epidemiologic studies (11 of 24) did not appear in the screening questionnaires, such as sociodemographic factors (e.g., education or occupation) and personal history (e.g., primary care visits, chest infections, previous diagnosis of asthma).

Seven factors unique to the epidemiologic literature 


\section{Table 2. Performance Characteristics of COPD Case-finding/Screening Tools by Setting}

\begin{tabular}{|c|c|c|c|c|c|c|c|c|c|c|c|c|c|}
\hline $\begin{array}{l}\text { Study } \\
\text { Author }\end{array}$ & Title & Year & $\begin{array}{l}\text { Popu- } \\
\text { lation }\end{array}$ & $\begin{array}{l}\text { Name of } \\
\text { Screening } \\
\text { Instrument }\end{array}$ & $\begin{array}{c}\text { No. } \\
\text { of } \\
\text { Items }\end{array}$ & $\begin{array}{l}\text { Score } \\
\text { Range }\end{array}$ & $\begin{array}{l}\text { Significant } \\
\text { Cut-point }\end{array}$ & Sensitivity & Specificity & $\begin{array}{c}\text { Positive } \\
\text { Predictive } \\
\text { Value }\end{array}$ & $\begin{array}{c}\text { Negative } \\
\text { Predictive } \\
\text { Value }\end{array}$ & $\begin{array}{l}\text { Likelihood } \\
\text { Ratio LR } \\
(95 \% \mathrm{Cl})\end{array}$ & $\begin{array}{c}\text { Area } \\
\text { Under the } \\
\text { ROC } \\
\text { Curve }\end{array}$ \\
\hline Calverley ${ }^{16}$ & $\begin{array}{l}\text { Development } \\
\text { of a population- } \\
\text { based screening } \\
\text { questionnaire } \\
\text { for COPD }\end{array}$ & 2005 & $\begin{array}{c}\text { Gen- } \\
\text { eral } \\
\text { popu- } \\
\text { lation }\end{array}$ & $\begin{array}{l}\text { "Could } \\
\text { it be } \\
\text { cOPD" }\end{array}$ & 5 & $0-5$ & $\begin{array}{l}3 \text { points, } \\
\text { age }>35\end{array}$ & $80.8 \%$ & $62.8 \%$ & $32.7 \%$ & $93.6 \%$ & - & -- \\
\hline Raghavan 17 & $\begin{array}{l}\text { Components of } \\
\text { the COPD } \\
\text { Assessment } \\
\text { Test associated } \\
\text { with a diagnosis } \\
\text { of COPD in a } \\
\text { random } \\
\text { population } \\
\text { sample }\end{array}$ & 2012 & $\begin{array}{l}\text { Gen- } \\
\text { eral } \\
\text { popu- } \\
\text { lation }\end{array}$ & $\begin{array}{c}\text { CAT } \\
\text { question- } \\
\text { naire } \\
\text { item } \\
\text { breath- } \\
\text { lessness } \\
(0-5) / \\
\text { smoking } \\
(0,1,2) / \\
\text { age }(0,1)\end{array}$ & 3 & $0-40$ & $\begin{array}{c}\text { Decision } \\
\text { tree } \\
\text { screening } \\
\text { model } \\
\text { ( } \geq 55 \\
\text { years; } \\
\text { Current } \\
\text { smoker; } \\
\text { breath- } \\
\text { lessness } \\
\text { of 4) }\end{array}$ & $77.6 \%$ & $64.9 \%$ & $18.6 \%$ & $96.5 \%$ & 2.21 & 0.772 \\
\hline van Schayck ${ }^{18}$ & $\begin{array}{l}\text { Comparison } \\
\text { of existing } \\
\text { symptom-based } \\
\text { questionnaires } \\
\text { for identifying } \\
\text { COPD in the } \\
\text { general practice } \\
\text { setting }\end{array}$ & 2005 & $\begin{array}{l}\text { Gen- } \\
\text { eral } \\
\text { popu- } \\
\text { lation }\end{array}$ & $\begin{array}{c}\text { Case- } \\
\text { finding } \\
\text { tool }\end{array}$ & 7 & -- & -- & $71.0 \%$ & $67.0 \%$ & $25.0 \%$ & $94.0 \%$ & -- & 0.747 \\
\hline Yawn 19 & $\begin{array}{l}\text { Development of } \\
\text { the Lung Function } \\
\text { Questionnaire } \\
\text { to identify } \\
\text { airflow obstruction }\end{array}$ & 2010 & $\begin{array}{l}\text { Gen- } \\
\text { eral } \\
\text { popu- } \\
\text { lation }\end{array}$ & $\begin{array}{l}\text { Lung } \\
\text { Function } \\
\text { Question- } \\
\text { naire } \\
\text { (LFQ) }\end{array}$ & 5 & 25 & $\begin{array}{c}\geq 3 \\
\text { questions }\end{array}$ & $77.8 \%$ & $52.4 \%$ & -- & - & - & 0.651 \\
\hline $\mathrm{Kotz}^{20}$ & $\begin{array}{c}\text { External validation } \\
\text { of a COPD } \\
\text { diagnostic } \\
\text { questionnaire }\end{array}$ & 2008 & $\begin{array}{c}\text { Gen- } \\
\text { eral } \\
\text { popu- } \\
\text { lation }\end{array}$ & $\begin{array}{c}\text { COPD } \\
\text { Diagnostic } \\
\text { Question- } \\
\text { naire } \\
\text { (CDQ) }\end{array}$ & 8 & $0-38$ & 19.5 & $65.8 \%$ & $54.0 \%$ & -- & - & -- & 0.65 \\
\hline Nelson $^{21}$ & $\begin{array}{l}\text { Questionnaires } \\
\text { and pocket } \\
\text { spirometers } \\
\text { provide an } \\
\text { alternative } \\
\text { approach for } \\
\text { COPD screening } \\
\text { in the general } \\
\text { population }\end{array}$ & 2012 & $\begin{array}{c}\text { Gen- } \\
\text { eral } \\
\text { popu- } \\
\text { lation }\end{array}$ & $\begin{array}{c}\text { No } \\
\text { name }\end{array}$ & 7 & -- & -- & $40.7 \%$ & $97.7 \%$ & -- & -- & - & -- \\
\hline Zhou $^{22}$ & $\begin{array}{l}\text { Development } \\
\text { and validation } \\
\text { of a chronic } \\
\text { obstructive } \\
\text { pulmonary } \\
\text { disease screening } \\
\text { questionnaire } \\
\text { in China }\end{array}$ & 2013 & $\begin{array}{l}\text { Gen- } \\
\text { eral } \\
\text { popu- } \\
\text { lation }\end{array}$ & $\begin{array}{l}\text { COPD- } \\
\text { Screening } \\
\text { Question- } \\
\text { naire } \\
\text { (COPD- } \\
\text { SQ) }\end{array}$ & 7 & - & $\geq 16$ & $60.6 \%$ & $85.2 \%$ & $35.1 \%$ & $95.0 \%$ & 4.09 & 0.812 \\
\hline
\end{tabular}




\begin{tabular}{|c|c|c|c|c|c|c|c|c|c|c|c|c|c|}
\hline $\begin{array}{l}\text { Study } \\
\text { Author }\end{array}$ & Title & Year & $\begin{array}{l}\text { Popu- } \\
\text { lation }\end{array}$ & $\begin{array}{l}\text { Name of } \\
\text { Screening } \\
\text { Instrument It }\end{array}$ & $\begin{array}{c}\text { No. } \\
\text { of } \\
\text { Items }\end{array}$ & $\begin{array}{l}\text { Score } \\
\text { Range }\end{array}$ & $\begin{array}{l}\text { Significant } \\
\text { Cut-point }\end{array}$ & Sensitivity & Specificity & $\begin{array}{l}\text { Positive } \\
\text { Predictive } \\
\text { Value }\end{array}$ & $\begin{array}{c}\text { Negative } \\
\text { Predictive } \\
\text { Value }\end{array}$ & $\begin{array}{l}\text { Likelihood } \\
\text { Ratio LR } \\
(95 \% \text { Cl) }\end{array}$ & $\begin{array}{c}\text { Area } \\
\text { Under the } \\
\text { ROC } \\
\text { Curve }\end{array}$ \\
\hline $\begin{array}{l}\text { Franco- } \\
\text { Marina }^{23}\end{array}$ & $\begin{array}{l}\text { Efficient screening } \\
\text { for COPD using } \\
\text { three steps: a } \\
\text { cross-sectional } \\
\text { study in } \\
\text { Mexico City }\end{array}$ & 2014 & $\begin{array}{c}\text { Gen- } \\
\text { eral } \\
\text { popu- } \\
\text { lation }\end{array}$ & $\begin{array}{l}\text { COPD } \\
\text { Scale }\end{array}$ & 2 & -- & $\geq 10$ & $91.7 \%$ & $46.7 \%$ & -- & -- & -- & 0.71 \\
\hline Kawayama $^{24}$ & $\begin{array}{l}\text { Validation of } \\
\text { symptom-based } \\
\text { COPD } \\
\text { questionnaires } \\
\text { in Japanese } \\
\text { subjects }\end{array}$ & 2008 & $\begin{array}{l}\text { Gen- } \\
\text { eral } \\
\text { popu- } \\
\text { lation }\end{array}$ & $\mathrm{CDQ}$ & 8 & $0-38$ & 19.5 & $84.8 \%$ & $64.7 \%$ & -- & -- & -- & 0.791 \\
\hline Freeman $^{25}$ & $\begin{array}{l}\text { Questions for } \\
\text { COPD diagnostic } \\
\text { screening in a } \\
\text { primary care } \\
\text { setting }\end{array}$ & 2005 & $\begin{array}{c}\text { Pri- } \\
\text { mary } \\
\text { care }\end{array}$ & $\begin{array}{c}\text { Model } \\
2\end{array}$ & 6 & $0-40$ & -- & $87.1 \%$ & $71.3 \%$ & $38.0 \%$ & $96.5 \%$ & 3.6 & 0.85 \\
\hline Frith 26 & $\begin{array}{l}\text { Simplified COPD } \\
\text { screening: validation } \\
\text { of the PiKo- } 6 \text { } \\
\text { in primary care }\end{array}$ & 2011 & $\begin{array}{l}\text { Pri- } \\
\text { mary } \\
\text { care }\end{array}$ & $\mathrm{CDQ}$ & 8 & -- & 19.5 & $71.0 \%$ & $62.0 \%$ & $42.0 \%$ & $85.0 \%$ & 1.88 & 0.72 \\
\hline Hanania $^{27}$ & $\begin{array}{l}\text { Predicting risk } \\
\text { of airflow } \\
\text { obstruction in } \\
\text { primary care: } \\
\text { validation of } \\
\text { the Lung Function } \\
\text { Questionnaire } \\
\text { (LFQ) }\end{array}$ & 2010 & $\begin{array}{c}\text { Pri- } \\
\text { mary } \\
\text { care }\end{array}$ & LFQ & 5 & 25 & $\leq 18$ & $82.6 \%$ & $47.8 \%$ & $26.5 \%$ & $92.3 \%$ & 1.58 & 0.652 \\
\hline Mintz $^{28}$ & $\begin{array}{l}\text { Prevalence of } \\
\text { airway obstruction } \\
\text { assessed by } \\
\text { Lung Function } \\
\text { Questionnaire }\end{array}$ & 2011 & $\begin{array}{c}\text { Pri- } \\
\text { mary } \\
\text { care }\end{array}$ & $\mathrm{LFQ}$ & 5 & 25 & $\leq 18$ & $88.0 \%$ & $25.0 \%$ & $21.0 \%$ & $90.0 \%$ & -- & -- \\
\hline Sichletidis 29 & $\begin{array}{l}\text { A combination } \\
\text { of the IPAG } \\
\text { questionnaire } \\
\text { and PiKo-6 flow } \\
\text { meter is a valuable } \\
\text { screening tool for } \\
\text { COPD in the } \\
\text { primary care } \\
\text { setting }\end{array}$ & 2011 & $\begin{array}{c}\text { Pri- } \\
\text { mary } \\
\text { care }\end{array}$ & $\begin{array}{c}\text { IPAG } \\
\text { question- } \\
\text { naire }\end{array}$ & 8 & $0-38$ & 19 & $72.0 \%$ & $77.0 \%$ & -- & -- & -- & -- \\
\hline Price ${ }^{30, a}$ & $\begin{array}{l}\text { Scoring system } \\
\text { and clinical } \\
\text { application of } \\
\text { COPD diagnostic } \\
\text { questionnaires }\end{array}$ & 2006 & $\begin{array}{c}\text { Pri- } \\
\text { mary } \\
\text { care }\end{array}$ & $\mathrm{CDQ}$ & 8 & $0-38$ & $\begin{array}{l}16.5 \\
19.5\end{array}$ & $\begin{array}{l}58.7 \% \\
80.4 \%\end{array}$ & $\begin{array}{l}77.0 \% \\
57.5 \%\end{array}$ & $\begin{array}{l}37.0 \% \\
30.3 \%\end{array}$ & $\begin{array}{l}89.0 \% \\
92.7 \%\end{array}$ & $\begin{array}{l}-- \\
--\end{array}$ & $\begin{array}{l}-- \\
--\end{array}$ \\
\hline Price $14, b$ & $\begin{array}{l}\text { Symptom-based } \\
\text { questionnaire } \\
\text { for identifying } \\
\text { chronic obstructive } \\
\text { pulmonary disease } \\
\text { in smokers }\end{array}$ & 2006 & $\begin{array}{c}\text { Pri- } \\
\text { mary } \\
\text { care }\end{array}$ & $\mathrm{CDQ}$ & 8 & $\mathrm{~N} / \mathrm{A}$ & $\begin{array}{l}\text { Inflec- } \\
\text { tion } \\
\text { point }\end{array}$ & $80.4 \%$ & $72.0 \%$ & -- & -- & -- & 0.816 \\
\hline
\end{tabular}




\begin{tabular}{|c|c|c|c|c|c|c|c|c|c|c|c|c|c|}
\hline $\begin{array}{l}\text { Study } \\
\text { Author }\end{array}$ & Title & Year & $\begin{array}{l}\text { Popu- } \\
\text { lation }\end{array}$ & $\begin{array}{l}\text { Name of } \\
\text { Screening } \\
\text { Instrument }\end{array}$ & $\begin{array}{c}\text { No. } \\
\text { of } \\
\text { Items }\end{array}$ & $\begin{array}{l}\text { Score } \\
\text { Range }\end{array}$ & $\begin{array}{l}\text { Significant } \\
\text { Cut-point }\end{array}$ & Sensitivity & Specificity & $\begin{array}{c}\text { Positive } \\
\text { Predictive } \\
\text { Value }\end{array}$ & $\begin{array}{c}\text { Negative } \\
\text { Predictive } \\
\text { Value }\end{array}$ & $\begin{array}{l}\text { Likelihood } \\
\text { Ratio LR } \\
(95 \% \mathrm{Cl})\end{array}$ & $\begin{array}{c}\text { Area } \\
\text { Under the } \\
\text { ROC } \\
\text { Curve }\end{array}$ \\
\hline Estes 31 & $\begin{array}{l}\text { An evidence- } \\
\text { based quality } \\
\text { improvement } \\
\text { perspective for a } \\
\text { chronic obstructive } \\
\text { pulmonary disease } \\
\text { case-finding } \\
\text { program }\end{array}$ & 2014 & $\begin{array}{c}\text { Pri- } \\
\text { mary } \\
\text { care }\end{array}$ & $\begin{array}{c}\text { COPD } \\
\text { Question- } \\
\text { naire }\end{array}$ & 9 & $0-38$ & $\geq 17$ & $50.0 \%$ & $71.0 \%$ & $17.0 \%$ & $93.0 \%$ & -- & 0.67 \\
\hline Stanley 32 & $\begin{array}{l}\text { Validation of } \\
\text { the COPD } \\
\text { Diagnostic } \\
\text { Questionnaire } \\
\text { in an Australian } \\
\text { general practice } \\
\text { cohort: a cross- } \\
\text { sectional study }\end{array}$ & 2014 & $\begin{array}{c}\text { Pri- } \\
\text { mary } \\
\text { care }\end{array}$ & $\mathrm{CDQ}$ & 8 & $0-38$ & 19.5 & $63.0 \%$ & $70.1 \%$ & $24.1 \%$ & $92.6 \%$ & -- & $\begin{array}{l}\mathrm{AUC}= \\
0.713\end{array}$ \\
\hline Martinez ${ }^{33}$ & $\begin{array}{l}\text { Development and } \\
\text { initial validation } \\
\text { of a self-scored } \\
\text { COPD Population } \\
\text { Screener } \\
\text { Questionnaire } \\
\text { (COPD-PS) }\end{array}$ & 2008 & $\begin{array}{l}\text { Pri- } \\
\text { mary } \\
\text { care } \\
\text { and } \\
\text { pulm- } \\
\text { onary } \\
\text { spec- } \\
\text { ialist }\end{array}$ & $\begin{array}{l}\text { COPD- } \\
\text { PS } \\
\text { question- } \\
\text { naire }\end{array}$ & 5 & $0-10$ & 5 & $84.4 \%$ & $60.7 \%$ & $56.8 \%$ & $86.4 \%$ & -- & $\begin{array}{c}\mathrm{AUC}= \\
0.73\end{array}$ \\
\hline Zarowitz $^{34}$ & $\begin{array}{l}\text { Development and } \\
\text { validation of a } \\
\text { screening tool } \\
\text { for chronic } \\
\text { obstructive } \\
\text { pulmonary } \\
\text { disease in } \\
\text { nursing home } \\
\text { residents }\end{array}$ & 2011 & $\begin{array}{c}\text { Spec- } \\
\text { ialist: } \\
\text { nur- } \\
\text { sing } \\
\text { home }\end{array}$ & $\begin{array}{c}\text { No } \\
\text { name }\end{array}$ & 4 & & $\begin{array}{l}\text { Yes to } \\
\text { at least } \\
\text { one and } \\
\text { if the only } \\
\text { one is } \\
\text { smoking } \\
\text { it must } \\
\text { be } \geq 19 \\
\text { pack } \\
\text { years }\end{array}$ & $90.6 \%$ & $77.8 \%$ & & & & $\begin{array}{l}\mathrm{ROC}= \\
0.903\end{array}$ \\
\hline Müllerová 35 & $\begin{array}{l}\text { Validation of } \\
\text { a chronic } \\
\text { obstructive } \\
\text { pulmonary } \\
\text { disease screening } \\
\text { questionnaire } \\
\text { for population } \\
\text { surveys }\end{array}$ & 2004 & $\begin{array}{l}\text { Spec- } \\
\text { ialist: } \\
\text { pulm- } \\
\text { onary } \\
\text { hos- } \\
\text { pital }\end{array}$ & $\begin{array}{l}\text { Confr- } \\
\text { onting } \\
\text { COPD } \\
\text { Survey } \\
\text { (per- } \\
\text { sonal } \\
\text { level } \\
\text { screener } \\
\text { for } \\
\text { COPD) }\end{array}$ & 5 & & -- & $92.0 \%$ & $79.4 \%$ & $92.0 \%$ & $79.3 \%$ & & $\begin{array}{c}\text { Chance- } \\
\text { corrected } \\
\text { agreement } \\
\text { k was } \\
0.66 \\
\text { (SE 0.098) }\end{array}$ \\
\hline
\end{tabular}

\footnotetext{
${ }^{a}$ The Price et al 2006 Chest article did not report 1 final recommended cut-point for their screener; hence the table presents the performance characteristics from the 2 cut-points presented in the article.

${ }^{\mathrm{b}}$ The Price et al 2006 Respiration article details the development of the COPD screening instrument and used a different sample of individuals. The screening instrument was validated in the Price 2006 Chest study.
} 


\section{Table 3. Predictive Factors Assessed in COPD Epidemiological Studies}

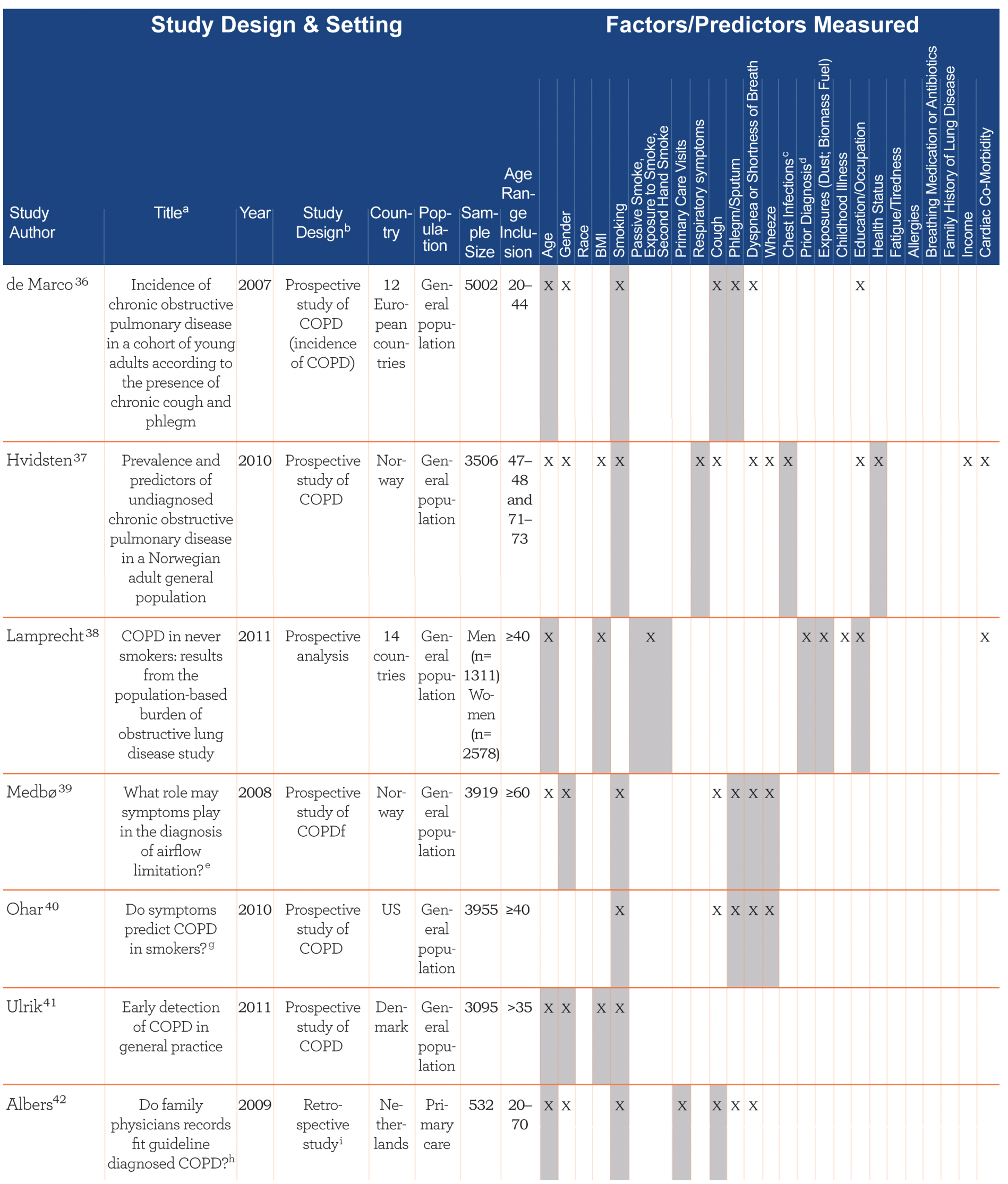




\begin{tabular}{|c|c|c|c|c|c|c|c|c|c|c|c|c|c|c|c|c|c|c|c|c|c|c|c|c|}
\hline & Study D & )esic & In \& Sett & ting & & & & & & & & ctor & $\mathrm{rs} / \mathrm{P}$ & Preo & dict & tor & $S$ & & eas & sur & rec & & & \\
\hline $\begin{array}{l}\text { Study } \\
\text { Author }\end{array}$ & Title $^{a}$ & Year & $\begin{array}{c}\text { Study } \\
\text { Design }^{\mathrm{b}}\end{array}$ & $\begin{array}{l}\text { Coun- } \\
\text { try }\end{array}$ & $\begin{array}{l}\text { Pop- } \\
\text { ula- } \\
\text { tion }\end{array}$ & $\begin{array}{l}\text { Sam- } \\
\text { ple } \\
\text { Size }\end{array}$ & $\begin{array}{l}\text { Age } \\
\text { Ran- } \\
\text { ge } \\
\text { Inclu- } \\
\text { sion }\end{array}$ & 这 & & 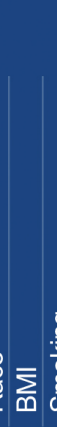 & & 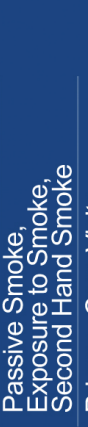 & 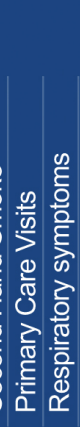 & 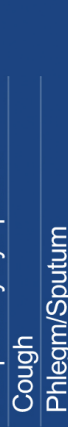 & 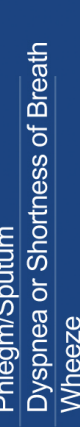 & 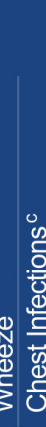 & 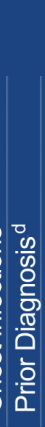 & 5 & 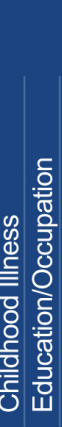 & 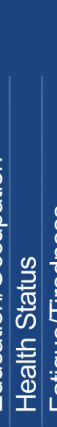 & 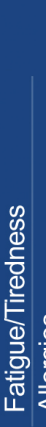 & 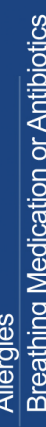 & 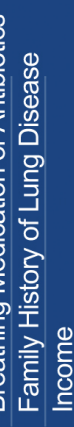 & 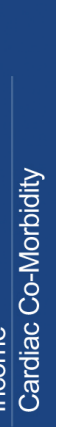 \\
\hline Hill $^{43}$ & $\begin{array}{c}\text { Prevalence and } \\
\text { underdiagnosis of } \\
\text { chronic obstructive } \\
\text { pulmonary disease } \\
\text { among patients } \\
\text { at risk in } \\
\text { primary care }\end{array}$ & 2010 & $\begin{array}{l}\text { Prospective } \\
\text { study of } \\
\text { COPD }\end{array}$ & $\begin{array}{l}\text { On- } \\
\text { tario, } \\
\text { Can- } \\
\text { ada }\end{array}$ & $\begin{array}{l}\text { Pri- } \\
\text { mary } \\
\text { care }\end{array}$ & 1003 & $\geq 40$ & $\mathrm{XX}$ & $\mathrm{x} x$ & & $\mathrm{X}$ & & $\mathrm{XX}$ & & & & & & & & & & & \\
\hline Løkke $e^{44}$ & $\begin{array}{l}\text { Detection of } \\
\text { previously } \\
\text { undiagnosed } \\
\text { cases of COPD } \\
\text { in a high-risk } \\
\text { population } \\
\text { identified in } \\
\text { general practice }\end{array}$ & 2012 & $\begin{array}{c}\text { Prospective } \\
\text { study of } \\
\text { COPD }\end{array}$ & $\begin{array}{l}\text { Den- } \\
\text { mark }\end{array}$ & $\begin{array}{l}\text { Pri- } \\
\text { mary } \\
\text { care }\end{array}$ & 4049 & $>35$ & X X & & $\mathrm{X}$ & $X$ & & & $X X$ & $X \times X$ & $x x$ & & & & & & & & \\
\hline Minas $^{45}$ & $\begin{array}{l}\text { COPD prevalence } \\
\text { and the differences } \\
\text { between newly } \\
\text { and previously } \\
\text { diagnosed COPD } \\
\text { patients in a } \\
\text { spirometry program }\end{array}$ & 2010 & $\begin{array}{l}\text { Prospective } \\
\text { study of } \\
\text { COPD }\end{array}$ & $\begin{array}{c}\text { Gree- } \\
\text { ce }\end{array}$ & $\begin{array}{l}\text { Pri- } \\
\text { mary } \\
\text { care }\end{array}$ & 1526 & $>30$ & $\mathrm{Xx}$ & & $\mathrm{X}$ & $\mathrm{X}$ & & & $\mathrm{X} x$ & $\mathrm{X} \times \mathrm{x}$ & & & $\mathrm{X}$ & X X & & & X & & \\
\hline $\begin{array}{l}\text { Van } \\
\text { Schayck }^{46}\end{array}$ & $\begin{array}{l}\text { Detecting patients } \\
\text { at a high risk of } \\
\text { developing chronic } \\
\text { obstructive pulmonary } \\
\text { disease in general } \\
\text { practice: cross- } \\
\text { sectional case } \\
\text { finding study }{ }^{j}\end{array}$ & 2002 & $\begin{array}{c}\text { Prospective } \\
\text { study }\end{array}$ & $\begin{array}{l}\mathrm{Ne}^{-} \\
\text {ther- } \\
\text { lands }\end{array}$ & $\begin{array}{l}\text { Pri- } \\
\text { mary } \\
\text { care }\end{array}$ & 651 & $\begin{array}{c}35- \\
70\end{array}$ & & & & & & & X & $\mathrm{XX}$ & & $\mathrm{x}$ & & & & $\mathrm{x}$ & $\mathrm{x}$ & $\mathrm{x}$ & \\
\hline $\begin{array}{l}\text { Vande- } \\
\text { voorde }\end{array}$ & $\begin{array}{l}\text { Early detection of } \\
\text { COPD: A case } \\
\text { finding study in } \\
\text { general practice }\end{array}$ & 2007 & $\begin{array}{l}\text { Prospective } \\
\text { study of } \\
\text { COPD }\end{array}$ & $\begin{array}{l}\text { Ne- } \\
\text { ther- } \\
\text { lands }\end{array}$ & $\begin{array}{l}\text { Pri- } \\
\text { mary } \\
\text { care }\end{array}$ & 146 & $\begin{array}{l}40- \\
70\end{array}$ & $\mathrm{X} X$ & & & $\mathrm{X}$ & & & $\mathrm{X}$ & $\mathrm{Xx}$ & & $\mathrm{x}$ & $X$ & & & $\mathrm{x}$ & & & \\
\hline
\end{tabular}

\footnotetext{
${ }^{\mathrm{a}} \mathrm{COPD}$ case definition unless otherwise noted was defined as postbronchodialator FEV1/FVC ratio $<0.70$

${ }^{b}$ Multivariate analysis used to assess significance in all studies unless otherwise noted

${ }^{c}$ Chest infections including cough, colds, respiratory issues that brought patient to the doctor

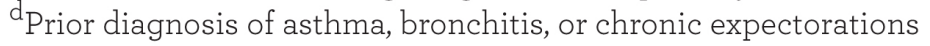

${ }^{e} \mathrm{COPD}$ case definition: Pre bronchodilator FEV1/FVC $<0.70$ for individuals 69 years and younger and pre bronchodilator

$\mathrm{FEV}_{1} / \mathrm{FVC}<0.65$ for individuals 70 years and older

${ }^{\mathrm{f}}$ Binary logistic regression was used in calculating odds ratios

${ }^{9} \mathrm{COPD}$ case definition: Post bronchodilator $\mathrm{FEV}_{1} / \mathrm{FVC}<70 \%$, aged $\geq 40$, and $\geq 20$ pack years of smoking

${ }^{h}$ COPD case definition: No FEV 1 reversibility $\geq 12 \%$ and $\geq 200 \mathrm{ml}$ after taking salbutamol

${ }^{i}$ Data source: DMCA, Detection, Intervention and Monitoring of COPD and Asthma

${ }^{j}$ COPD case definition: $\mathrm{FEV}_{1}<80 \%$
} 


\section{Table 4. Variables Used to Identify Cases of COPD by Study Type}

\begin{tabular}{|c|c|c|}
\hline Theme & Variables Used in Screening Tools & Variables Tested in Epidemiologic St \\
\hline Exposure & $\begin{array}{l}\text { Smoking status } \\
\text { Pack years }\end{array}$ & $\begin{array}{l}\text { Smoking status } \\
\text { Passive smoke, exposure to smokea, second hand smoke } \\
\text { Exposure to dust, biomass fuel, or other inhalant }\end{array}$ \\
\hline $\begin{array}{l}\text { Personal and } \\
\text { Family History }\end{array}$ & $\begin{array}{l}\text { Allergies } \\
\text { Prior diagnosis of asthma } \\
\text { Breathing-related hospitalizations }\end{array}$ & $\begin{array}{l}\text { Allergies } \\
\text { Prior diagnosis of asthma, bronchitis, or chronic } \\
\text { expectoration } \\
\text { Chest infections }{ }^{a} \\
\text { Childhood illness } \\
\text { Family history of lung disease } \\
\text { Frequency of primary care visits } \\
\text { Breathing medication or antibiotics } \\
\text { Cardiac co-morbidity }\end{array}$ \\
\hline Symptoms & $\begin{array}{l}\text { Cough } \\
\text { Phlegm } \\
\text { SOB } \\
\text { Wheeze }\end{array}$ & $\begin{array}{l}\text { Cough } \\
\text { Phlegm } \\
\text { SOB } \\
\text { Wheeze } \\
\text { Respiratory symptoms (combination) } \\
\text { Fatigue/tiredness }\end{array}$ \\
\hline Impact & $\begin{array}{l}\text { Activity limitation } \\
\text { (Breathing-related) }\end{array}$ & Self-rated health status ${ }^{a}$ \\
\hline Demographics & $\begin{array}{l}\text { Age } \\
\text { BMI }\end{array}$ & $\begin{array}{l}\text { Age } \\
\text { BMI } \\
\text { Gender } \\
\text { Race } \\
\text { Education/occupation } \\
\text { Income }\end{array}$ \\
\hline
\end{tabular}

\footnotetext{
${ }^{a}$ Significant independent predictor of COPD status

Variables in italics are unique to that study type.
}

were significant predictors of COPD diagnosis. These were chest infections ( $\mathrm{n}=1$ of 2 studies), childhood illness ( $n=1$ of 2 studies), frequency of primary care visits ( $n=1$ of 2 studies), respiratory symptoms ( $n=2$ of 2 studies), self-rated health status ( $n=1$ of 1 study), gender ( $\mathrm{n}=4$ of 9 studies, with male gender a significant predictor of COPD status), and education/occupation ( $n=1$ of 4 studies). Interestingly, 1 study $^{38}$ found exposure to organic dust and lower education were significant predictors of COPD in women who never smoked ( $n=1$ of 1 study); passive smoke exposure was also of borderline significance in this group. Factors not included in the screeners and not statistically significant in the epidemiological studies were income, fatigue/ tiredness, cardiac co-morbidity, and taking breathing medications or antibiotics.

\section{Discussion}

This paper summarizes predictive factors for identifying new cases of COPD (defined as the presence of airflow limitation on spirometry) using literature from casefinding/screening questionnaires and epidemiologic studies. The intent was to improve our understanding of the factors and sets of factors that can be used to identify people with undiagnosed COPD. This information is not only useful for general case-finding in clinical practice, but will be used together with data mining techniques and qualitative research methods to inform the development of candidate variables for a new method for identifying undiagnosed, clinicallysignificant COPD in primary care.

While the review summarized the COPD screening 
and epidemiologic studies separately, it is clear that, in many instances, COPD screening tools were built based upon the existing epidemiologic literature. For example, Calverley et $\mathrm{l}^{16}$ first identified characteristics of COPD patients by conducting a literature review, and then identified the correspondence to those specific questions in the NHANES III dataset. Similarly, van Schayk et al ${ }^{18}$ conducted a literature review to first identify previously available tools and potentially useful items for screening, and then retrospectively validated the selected set of items using the NHANES III dataset. Yawn et $\mathrm{al}^{19}$ used NHANES III data for empirical item selection and item-reduction, and conducted a qualitative assessment phase to assess the content and face validity of their items via physician focus groups and patient interviews. Martinez et $\mathrm{al}^{33}$ used a combination of expert opinion and patient survey data.

Not surprisingly, there was substantial overlap between the screening and epidemiologic studies amongst individual factors most likely to identify COPD. In both studies, factors that appear to be strongly associated with COPD were older age, BMI, smoking history, and symptoms such as cough, wheeze, and dyspnea. However, we also identified factors through the epidemiological studies that were not included in the screening studies. One of these factors was childhood illness. Other lines of research suggest that factors such as prenatal maternal smoking, low birth weight, postnatal tobacco exposure, and childhood respiratory infections may predispose individuals to the development of COPD. ${ }^{48}$ Therefore, it makes sense that factors like childhood illness might increase the likelihood of identifying individuals with COPD. History of chest infections, frequency of primary care visits, and poor self-rated health status were also factors identified in the epidemiologic studies but not tested in the screening studies. While it is possible that chest infections could predispose the development of COPD, it is also possible that chest infections, frequent primary care utilization, and poorer health status are manifestations of COPD, which could aid in the identification of high-risk COPD.

With respect to performance characteristics, it should be noted that the screening tools were tested in a variety of populations, which significantly influenced the sensitivity and specificity of the instruments. Several authors commented on how a study population can influence the performance properties of a screening tool. For example, the CDQ screening tool developed by Price et $\mathrm{al}^{30}$ had good performance properties in the initial evaluation ( $\mathrm{AUC}=0.82$ ). However, in 2 subsequent articles where other authors tested the same screening tool in different samples, the performance characteristics of the screening tool were reduced ( $A U C=0.65$ in Kotz et $\mathrm{al}^{20}$; $\mathrm{AUC}=0.72$ in Frith et $\mathrm{al}^{26}$ ). Frith et $\mathrm{al}^{26}$ suggested that the difference in results may be due to differences in the demographic characteristics of the study populations (the individuals in the original study by Price et $\mathrm{al}^{30}$ were younger with lower cumulative cigarette consumption compared to Frith et $\mathrm{al}^{26}$ ). Frith concluded that the utility of screening questionnaires may be more limited outside the development and validation population. Alternatively, Yawn et al ${ }^{19}$ developed a screening tool to be useful across a broad spectrum of patient types and settings, rather than targeted to a particular population (e.g., smokers), as many of the other studies were. ${ }^{20,25,26,28-30}$ Yawn et $\mathrm{al}^{19}$ tested their items in both a general population and a chronic bronchitic population and found that items performed similarly. This highlights the importance of considering the target population during questionnaire development and initial validation.

\section{Limitations}

Several limitations of this review should be mentioned. First, due to the heterogeneity among studies in terms of study design, the large number of different risk factors studied, and the different ways in which certain variables were measured (e.g., smoking), the use of meta-analytic techniques was not the appropriate methodology to synthesize results. This may be possible in the future, as more studies utilize the same screening tool or capture the same risk factors in the same way. Second, the review was limited to studies published in English, and the majority of the studies were from developed countries, limiting generalizability of results to patients in other countries. In developing countries, risk factors such as biomass fuel exposure and occupational exposures are likely important and would need to be considered when developing a case-finding questionnaire specific to these countries. Finally, most studies use $\mathrm{FEV}_{1} / \mathrm{FVC}$ $<0.70$ as the cutoff for COPD diagnosis, which is likely to overdiagnose disease in older individuals.

\section{Implications for Practice and Further Research}

Previous research suggests that COPD is widely underdiagnosed, ${ }^{9}$ with many individuals experiencing symptoms of clinically-significant COPD or AECOPD, with diagnosis at the time of the event. Case-finding 
methods are needed to easily and accurately identify these patients as part of routine clinical practice. Screening tools are available which cover several key factors, e.g., the COPD Population Screener Questionnaire (COPD-PS) ${ }^{33}$ and the Lung Function Questionnaire (LFQ), ${ }^{19}$ but our literature review highlights new variables such as prenatal maternal smoking, low birth weight, postnatal tobacco exposure, and childhood respiratory infection that could further the precision of screening in primary care settings. Factors were identified in the epidemiologic studies that have not been tested in screening studies, including prior history of chest infections, childhood illness, selfrated health status, and frequency of primary care visits. Incorporating these missing variables into a COPD case-finding measure may allow clinicians to accurately identify not only undiagnosed cases of COPD, but also patients at risk of clinically-significant COPD that would benefit most from treatment. Currently, no method has been developed to identify this group in most need of care. The findings from the present study are currently being used in further work to find the smallest set or combination of items that are able to identify undiagnosed, high-risk COPD patients in primary care. The use of values from a peak flow meter, such as that used by Nelson et $\mathrm{al}^{21}$ and Sichletidis et al, ${ }^{29}$ combined with the questionaniare may further refine the patient population in whom spirometry is needed.

\section{Conclusion}

This review lays the groundwork for a multi-method development of a new screening approach for identifying undiagnosed, clinically-significant COPD in a primary care setting. Findings from this review highlight several factors that previous research has shown to be consistently and significantly associated with COPD case-finding in a variety of health care settings: age, BMI, wheeze, phlegm/sputum, smoking, cough, and dyspnea/breathlessness. However, our review suggests there are other factors-such as childhood illness and education/occupation-that are not included in existing screening questionnaires, but may also increase our ability to more precisely identify undiagnosed cases of clinically-significant COPD.

\section{Acknowledgements}

The authors thank Sarah Clifford and Lindsey Murray for their comments during the early stages of this work and Kathryn Miller for text editing and formatting.

\section{Declaration of Interest}

Dr. Han has consulted for GlaxoSmithKline, Boehringer-Ingelheim, and Regeneron. She has served on speaker bureaus for GlaxoSmithKline, Novartis, Boehringer-Ingelheim, Forest, and Grifols. Dr. Leidy, Dr. Bacci, Ms. Kim, Ms. Steenrod, and Ms. Murray are employees of Evidera, a health care research firm that provides consulting and other research services to pharmaceutical, device, government, and non-government organizations. In this salaried position, they work with a variety of companies and organizations and receive no payment or honoraria directly from these organizations for services rendered. Dr. Mannino has received honoraria/consulting fees and served on speaker bureaus for GlaxoSmithKline PLC, Novartis Pharmaceuticals, Pfizer Inc., BoehringerIngelheim, AstraZeneca PLC, Forest Laboratories Inc., Merck, Amgen, and Creative Educational Concepts. Furthermore, he has received royalties from UptoDate and is on the Board of Directors of the COPD Foundation. Dr. Thomashow has consulted for Boehringer-Ingelheim and has been on advisory boards for GlaxoSmithKline PLC, Novartis, AstraZeneca PLC, and Forest. Dr. Barr received grant funding for this work from the National Heart Lung and Blood Institute under R01HL114055. Dr. Barr received grant support from the National Institutes of Health (NIH), the United States Environmental Protection Agency, and the Alpha-1 Foundation; he has received royalties from UpToDate. Dr. Make has participated in research studies and/or served on medical advisory boards for AstraZeneca, BoehringerIngelheim, CSL Bering, GlaxoSmithKline, Forest, Novartis, Spiration, and Sunovion. Dr. Bowler's work has been funded by the NIH, FAMRI, Butcher Foundation, and John W. Carson Foundation. He participates in AstraZeneca and GlaxoSmithKline- sponsored clinical trials. He has received compensation as a member of scientific advisory boards of Boehringer Ingelheim Pharmaceutical. Dr. Rennard has had or currently has a number of relationships with companies who provide products and/or services relevant to outpatient management of chronic obstructive pulmonary disease, including the American Association of Respiratory Care, the American Board of Internal Medicine, Able Associates, Align2 Acton, Almirall, APT, AstraZeneca, the American Thoracic Society, Beilenson, Boehringer Ingelheim, Chiesi, CIPLA, Clarus Acuity, CME Incite, 
the COPD Foundation, Cory Paeth, CSA, CSL Behring, CTS Carmel, Dailchi Sankyo, Decision Resources, Dunn Group, Easton Associates, Elevation Pharma, FirstWord, Forest, GLG Research, Gilead, Globe Life Sciences, GlaxoSmithKline, Guidepoint, Health Advance, HealthStar, HSC Medical Education, Johnson and Johnson, Leerink Swan, LEK, McKinsey, Medical Knowledge, Medimmune, Merck, Navigant, Novartis, Nycomed, Osterman, Pearl, PeerVoice, Penn Technology, Pennside, Pfizer, Prescott, Pro Ed Communications, PriMed, Pulmatrix, Quadrant, Regeneron, Saatchi and Saatchi, Sankyo, Schering, Schlesinger Associates, Shaw Science, Strategic North, Summer Street Research, Synapse, Takeda, Telecon SC, ThinkEquity; these relationships include serving as a consultant, advising regarding clinical trials, speaking at continuing medical education programs and performing funded research both at basic and clinical levels. Dr. Rennard does not own any stock in any pharmaceutical companies. Dr. Houfek declares no conflict of interest. Dr. Yawn has received research funding from the NIH, AHRQ, the Centers for Disease Control and Prevention, and from Boehringer Ingelheim for research on COPD. Dr. Yawn has received compensation from Merck and Forrest for COPD advisory boards on COPD, and Grifols for advisory board on alpha-1 antitrypsin deficiency states. Catherine Meldrum declares no conflict of interest. John W. Walsh declares no conflict of interest. Dr. Martinez has participated in steering committees on COPD or idiopathic pulmonary fibrosis sponsored by Bayer, Centocor, Forest, Gilead, Janssens, GlaxoSmithKline, Nycomed/Takeda and Promedior. He has participated in advisory boards for COPD or IPF for Actelion, Amgen, Astra Zeneca, Boehringer Ingelheim, Carden Jennings, CSA Medixcal, Ikaria, Forest, Genentech, GlaxoSmithKline, Janssens, Merck, Pearl, Nycomed/ Takeda, Pfizer, Roche, Sudler \& Hennessey, Veracyte, and Vertex. He has prepared or presented continuing medical presentations on COPD or IPF for the American College of Chest Physicians, the American Thoracic Society, CME Incite, the Center for Health Care Education, Inova Health Systems, MedScape, Miller Medical, the National Association for Continuing Education, Paradigm, Peer Voice, Projects in Knowledge, Spectrum Health System, St. John's Hospital, St. Mary's Hospital, the University of Illinois Chicago, the University of Texas Southwestern, the University of Virginia, UpToDate, and Wayne State University. Dr. Martinez has participated in data safety monitoring committees sponsored by GlaxoSmithKline and Stromedix. He has assisted with U.S. Food and Drug Administration presentations sponsored by Boehringer Ingelheim, GlaxoSmithKline, and Ikaria. He has spoken on COPD for Bayer, Forest, GlaxoSmithKline, and Nycomed/Takeda. He has participated in advisory teleconferences sponsored by the American Institute for Research, Axon, Grey Healthcare, Johnson \& Johnson, and Merion. He has received book royalties from Informa. 


\section{References}

1. Global Initiative for Chronic Obstructive Lung Disease (GOLD). Global Strategy for the Diagnosis, Management, and Prevention of COPD. GOLD website. http://www.goldcopd.org/uploads/ users/files/GOLD_Report_2013Feb13.pdf. Accessed November 2014.

2. Espinosa de los Monteros MJ, Pena C, Soto Hurtado EJ, Jareno J, Miravitlles M. Variability of respiratory symptoms in severe COPD. Arch Bronconeumol. 2012;48(1):3-7. doi: http://dx.doi.org/10.1016/j.arbr.2011.07.006

3. Kessler R, Partridge MR, Miravitlles M, et al. Symptom variability in patients with severe COPD: a pan-European cross-sectional study. Eur Respir J. 2011;37(2):264-272. doi: http://dx.doi.org/10.1183/09031936.00051110

4. Brown DW, Croft JB, Greenlund KJ, Giles WH. Trends in hospitalization with chronic obstructive pulmonary diseaseUnited States, 1990-2005. COPD. 2010;7(1):59-62. doi: http://dx.doi.org/10.3109/15412550903499548

5. Centers for Disease Control and Prevention. Deaths from chronic obstructive pulmonary disease - United States 20002005. Morb Mortal Wkly Rep. 2008;57(45):1229-1232.

6. Kochanek KD, Xu J, Murphy SL, Minino AM, Kung HC. Deaths: final data for 2009. Natl Vital Stat Rep. 2011;60(3):1-117.

7. Centers for Disease Control and Prevention. Chronic obstructive pulmonary disease among adults - United States 2011. Morb Mortal Wkly Rep. 2012;61(46):938-943.

8. Mannino DM, Buist AS. Global burden of COPD: risk factors, prevalence, and future trends. Lancet. 2007;370(9589):765-773. doi: http://dx.doi.org/10.1016/S0140-6736(07)61380-4

9. Mannino DM, Gagnon RC, Petty TL, Lydick E. Obstructive lung disease and low lung function in adults in the United States: data from the National Health and Nutrition Examination Survey, 1988-1994. Arch Intern Med. 2000;160(11):1683-1689. doi: http://dx.doi.org/10.1001/archinte.160.11.1683

10. Roche N, Gaillat J, Garre M, et al. Acute respiratory illness as a trigger for detecting chronic bronchitis in adults at risk of COPD: a primary care survey. Prim Care Respir J. 2010;19(4):371377. doi: http://dx.doi.org/10.4104/pcri.2010.00042

11. Martinez FJ. A Case-finding strategy for moderate-to-severe COPD in the United States. Paper presented at: National Heart Lung and Blood Institute Workshop, 2009.

12. Wilt TJ, Niewoehner D, MacDonald R, Kane RL. Management of stable chronic obstructive pulmonary disease: a systematic review for a clinical practice guideline. Ann Intern Med. 2007;147(9):639-653. doi: http://dx.doi.org/10.7326/0003-4819147-9-200711060-00009

13. Qaseem A, Snow V, Shekelle P, et al. Diagnosis and management of stable chronic obstructive pulmonary disease: a clinical practice guideline from the American College of Physicians. Ann Intern Med. 2007;147(9):633-638. doi: http://dx.doi. org/10.7326/0003-4819-147-9-200711060-00008

14. Price DB, Tinkelman DG, Halbert RJ, et al. Symptom-based questionnaire for identifying COPD in smokers. Respiration. 2006;73(3):285-295. doi: http://dx.doi.org/10.1159/000090142
15. JonesPW,HardingG,BerryP,etal.Developmentandfirstvalidation of the COPD Assessment Test. Eur Respir J. 2009;34(3):648-654. doi: http://dx.doi.org/10.1183/09031936.00102509

16. Calverley PM, Nordyke RJ, Halbert RJ, Isonaka S, Nonikov D. Development of a population-based screening questionnaire for COPD. COPD. 2005;2(2):225-232. doi: http://dx.doi.org/10.1081/COPD-57594

17. Raghavan N, Lam YM, Webb KA, et al. Components of the COPD Assessment Test (CAT) associated with a diagnosis of COPD in a random population sample. COPD. 2012; 9(2):175183. doi: http://dx.doi.org/10.3109/15412555.2011.650802

18. van Schayck CP, Halbert RJ, Nordyke RJ, et al. Comparison of existing symptom-based questionnaires for identifying COPD in the general practice setting. Respirology. 2005;10(3):323-333. doi: http://dx.doi.org/10.1111/j.1440-1843.2005.00720.x

19. Yawn BP, Mapel DW, Mannino DM, et al. Development of the Lung Function Questionnaire (LFQ) to identify airflow obstruction. Int J Chron Obstruct Pulmon Dis. 2010;5:1-10.

20. Kotz D, Nelemans P, van Schayck CP, Wesseling GJ. External validation of a COPD diagnostic questionnaire. Eur Respir J. 2008;31(2):298-303. doi: http://dx.doi.org/10.1183/09031936.00074307

21. Nelson SB, LaVange LM, Nie Y, et al. Questionnaires and pocket spirometers provide an alternative approach for COPD screening in the general population. Chest. 2012;142(2):358-366. doi: http://dx.doi.org/10.1378/chest.11-1474

22. Zhou YM, Chen SY, Tian J, et al. Development and validation of a chronic obstructive pulmonary disease screening questionnaire in China. Int J Tuberc Lung Dis. 2013;17(12):1645-1651. doi: http://dx.doi.org/10.5588/ijtld.12.0995

23. Franco-Marina F, Fernandez-Plata R, Torre-Bouscoulet L, et al. Efficient screening for COPD using three steps: a crosssectional study in Mexico City. NPJ Prim Care Respir Med. 2014;24:14002. doi: http://dx.doi.org/10.1038/npjpcrm.2014.2

24. Kawayama T, Minakata Y, Matsunaga K, et al. Validation of symptom-based COPD questionnaires in Japanese subjects. Respirology. 2008;13(3):420-426. doi: http://dx.doi.org/10.1111/j.1440-1843.2008.01241.x

25. Freeman D, Nordyke RJ, Isonaka S, et al. Questions for COPD diagnostic screening in a primary care setting. Respir Med. 2005;99(10):1311-1318. doi: http://dx.doi.org/10.1016/j.rmed.2005.02.037

26. Frith P, Crockett A, Beilby J, et al. Simplified COPD screening: validation of the PiKo-6(R) in primary care. Prim Care Respir J. 2011;20(2):190-198. doi: http://dx.doi.org/10.4104/pcrj.2011.00040

27. Hanania NA, Mannino DM, Yawn BP, et al. Predicting risk of airflow obstruction in primary care: Validation of the lung function questionnaire (LFQ). Respir Med. 2010;104(8):11601170. doi: http://dx.doi.org/10.1016/j.rmed.2010.02.009

28. Mintz ML, Yawn BP, Mannino DM, et al. Prevalence of airway obstruction assessed by lung function questionnaire. Mayo Clin Proc. 2011;86(5):375-381. doi: http://dx.doi.org/10.4065/mcp.2010.0787 
29. Sichletidis L, Spyratos D, Papaioannou M, et al. A combination of the IPAG questionnaire and PiKo-6(R) flow meter is a valuable screening tool for COPD in the primary care setting. Prim Care Respir J. 2011;20(2):184-189.

doi: http://dx.doi.org/10.4104/pcrj.2011.00038

30. Price DB, Tinkelman DG, Nordyke RJ, et al. Scoring system and clinical application of COPD diagnostic questionnaires. Chest. 2006;129(6):1531-1539. doi: http://dx.doi.org/10.1378/chest.129.6.1531

31. Estes TS, Short N, Bowser D, Boyle A. An evidence-based quality improvement perspective for a chronic obstructive pulmonary disease case-finding program. Chron Respir Dis. 2014;11(3):131138. doi: http://dx.doi.org/10.1177/1479972314538979

32. Stanley AJ, Hasan I, Crockett AJ, van Schayck OC, Zwar NA. Validation of the COPD Diagnostic Questionnaire in an Australian general practice cohort: a cross-sectional study. Prim Care Respir J. 2014;23(1):92-97.

doi: http://dx.doi.org/10.4104/pcrj.2014.00015

33. Martinez FJ, Raczek AE, Seifer FD, et al. Development and initial validation of a self-scored COPD Population Screener Questionnaire (COPD-PS). COPD. 2008;5(2):85-95. doi: http://dx.doi.org/10.1080/15412550801940721

34. Zarowitz BJ, O'Shea T, Lefkovitz A, Peterson EL. Development and validation of a screening tool for chronic obstructive pulmonary disease in nursing home residents. J Am Med Dir Assoc. 2011;12(9):668-674. doi: http://dx.doi.org/10.1016/j.jamda.2010.11.007

35. Mullerova H, Wedzicha J, Soriano JB, Vestbo J. Validation of a chronic obstructive pulmonary disease screening questionnaire for population surveys. Respir Med. 2004;98(1):78-83. doi: http://dx.doi.org/10.1016/j.rmed.2003.08.009

36. de Marco R, Accordini S, Cerveri I, et al. Incidence of chronic obstructive pulmonary disease in a cohort of young adults according to the presence of chronic cough and phlegm. Am J Respir Crit Care Med. 2007;175(1):32-39.

doi: http://dx.doi.org/10.1164/rccm.200603-3810C

37. Hvidsten SC, Storesund L, Wentzel-Larsen T, Gulsvik A, Lehmann S. Prevalence and predictors of undiagnosed chronic obstructive pulmonary disease in a Norwegian adult general population. Clin Respir J. 2010;4(1):13-21. doi: http://dx.doi.org/10.1111/j.1752-699X.2009.00137.x

38. Lamprecht B, McBurnie MA, Vollmer WM, et al. COPD in never smokers: results from the population-based burden of obstructive lung disease study. Chest. 2011;139(4):752-763. doi: http://dx.doi.org/10.1378/chest.10-1253

39. Medbo A, Melbye $H$. What role may symptoms play in the diagnosis of airflow limitation? A study in an elderly population. Scand J Prim Health Care. 2008;26(2):92-98. doi: http://dx.doi.org/10.1080/02813430802028938

40. Ohar JA, Sadeghnejad A, Meyers DA, Donohue JF, Bleecker ER. Dosymptoms predict COPD in smokers? Chest.2010;137(6):13451353. doi: http://dx.doi.org/10.1378/chest.09-2681

41. Ulrik CS, Lokke A, Dahl R, et al. Early detection of COPD in general practice. Int J Chron Obstruct Pulmon Dis. 2011;6:123127. doi: http://dx.doi.org/10.2147/COPD.S16929
42. Albers M, Schermer T, Molema J, et al. Do family physicians' records fit guideline diagnosed COPD? Fam Pract. 2009;26(2):8187. doi: http://dx.doi.org/10.1093/fampra/cmp005

43. Hill K, Goldstein RS, Guyatt GH, et al. Prevalence and underdiagnosis of chronic obstructive pulmonary disease among patients at risk in primary care. CMAJ. 2010;182(7):673-678. doi: http://dx.doi.org/10.1503/cmaj.091784

44. Lokke A, Ulrik CS, Dahl R, et al. Detection of previously undiagnosed cases of COPD in a high-risk population identified in general practice. COPD. 2012;9(5):458-465. doi: http://dx.doi.org/10.3109/15412555.2012.685118

45. Minas M, Hatzoglou C, Karetsi E, et al. COPD prevalence and the differences between newly and previously diagnosed COPD patients in a spirometry program. Prim Care Respir J. 2010;19(4):363-370. doi: http://dx.doi.org/10.4104/pcrj.2010.00034

46. Schayck CPv, Loozen JMC, Wagena E, Akkermans RP, Wesseling GJ. Detecting patients at a high risk of developing chronic obstructive pulmonary disease in general practice: cross sectional case finding study. BMJ. 2002;324:1370. doi:http://dx.doi.org/10.1136/bmj.324.7350.1370

47. Vandevoorde J, Verbanck S, Gijssels L, et al. Early detection of COPD: a case finding study in general practice. Respir Med. 2007;101(3):525-530. doi: http://dx.doi.org/10.1016/j.rmed.2006.06.027

48. Stocks J, Sonnappa S. Early life influences on the development of chronic obstructive pulmonary disease. Ther Adv Respir Dis. 2013;7(3):161-173. doi: http://dx.doi.org/10.1177/1753465813479428 